Revista Universo Contábil, ISSN 1809-3337
Blumenau, v. 14, n. 3, p. 28-45, jul./set., 2018
doi:10.4270/ruc.2018318
Disponivel em www.furb.br/universocontabil

\title{
QUAIS EVENTOS CORPORATIVOS INFLUENCIAM OS RETORNOS DAS AÇÕES? UM ESTUDO BASEADO EM BOOTSTRAP1
}

\section{WHAT CORPORATE EVENTS INFLUENCE STOCK MARKET RETURNS? A BOOTSTRAP- BASED STUDY}

\section{¿QUÉ EVENTOS CORPORATIVOS AFECTAN RENTABILIDAD DE LAS ACCIONES? UN ESTUDIO BASADO EN BOOTSTRAP}

\author{
Rafael Moreira Antônio \\ Mestre em Controladoria e Finanças pela FEARP da Universidade de São Paulo \\ Doutorando em Controladoria e Contabilidade pela FEARP da Universidade de São Paulo \\ Endereço: Av. Bandeirantes, 3.900 - Monte Alegre \\ CEP: $14040-900$ - Ribeirão Preto - SP - Brasil \\ E-mail: rafaelmantonio@gmail.com \\ Telefone: (16) 3315-0206 \\ Ralph Melles Sticca \\ Mestre em Controladoria e Finanças pela FEA-RP da Universidade de São Paulo \\ Doutorando em Controladoria e Contabilidade pela FEA-RP da Universidade de São Paulo \\ Endereço: Av. Bandeirantes, 3.900 - Monte Alegre \\ CEP: $14040-900$ - Ribeirão Preto - SP - Brasil \\ E-mail: ralphsticca@usp.br \\ Telefone: (16) 3315-0206 \\ Marcelo Augusto Ambrozini \\ Doutor em Administração de Organizações FEA-RP da Universidade de São Paulo \\ Professor PPG em Controladoria e Contabilidade da FEA-RP da Universidade de São Paulo \\ Endereço: Av. Bandeirantes, 3.900 - Monte Alegre \\ CEP: $14040-900$ - Ribeirão Preto - SP - Brasil \\ E-mail: marceloambrozini@yahoo.com.br \\ Telefone: 016-99204-3775
}

\section{RESUMO}

O objetivo deste trabalho é verificar o impacto que determinados eventos corporativos causam no retorno das ações de empresas brasileiras, principalmente os relativos a divulgação de: a) grupamentos de ações; b) desdobramento de ações; c) distribuição de proventos (dividendos e $\mathrm{JCP}$ ); e d) alterações de ratings de crédito. Diferente dos estudos anteriores desenvolvidos no Brasil sobre o tema, o presente trabalho utiliza estudos de eventos baseado em Bootstrap, cujo

\footnotetext{
${ }^{1}$ Artigo recebido em 30/01/2017. Revisado por pares em 27/02/2019. Reformulado em 06/03/2019. Recomendado para publicação em 06/03/2019 por Tarcísio Pedro da Silva. Publicado em 30/03/2019. Organização responsável pelo periódico: FURB.
} 
principal benefício é o ajuste para pequenas amostras, como é o caso dos eventos coletados de alterações de ratings (com 274 observações), grupamentos (com 479 observações) e desdobramentos (com 306 observações), em comparação ao número de eventos de divulgações de dividendos e JCP (com 10.258 observações). O período mínimo de análise foi de dez anos, ou seja, de janeiro de 2006 a dezembro de 2015 e os resultados indicaram que as distribuições de dividendos e JCP influenciaram o mercado positivamente, já que o retorno anormal médio das ações foi de $0,70 \%$ no dia do evento. Os desdobramentos de ações também foram capazes de influenciar o mercado, já que no dia do evento o retorno médio anormal das ações foi de $1,96 \%$. Por sua vez, os grupamentos de ações não apresentaram retornos anormais estatisticamente significantes, mas pôde-se inferir graficamente que o retorno das ações em torno deste evento segue uma tendência de baixa.

Palavras-chave: Retorno de Ações; Ratings; Distribuição de Proventos; Grupamentos de Ações; Desdobramentos de Ações.

\section{ABSTRACT}

This study aims to verify the impact that certain corporate events cause on the return of Brazilian companies' shares, especially those related to the disclosure of a) inplits; b) splits; c) dividend payout and JCP; e d) changes in credit ratings. Different from the previous studies performed in Brazil on this research line, the present paper uses Bootstrap based on event studies. The main benefit of using this method is the adjustment for small samples, such as the events collected from changes in ratings (with 274 observations), inplits (with 479 observations) and splits (with 306 observations), compared to the number of dividend payout and JCP (with 10,258 observations). From a database of 11,317 events, the results showed that dividend and JCP distributions influenced the market positively, since the average abnormal stock return was $0.70 \%$ on the day of the event. The disclosure of splits was also able to influence the market, as on the day of the event the average abnormal stock return was $1.96 \%$. In turn, the inplits did not present abnormal returns statistically significant, but it was possible to infer graphically that the return of the shares around this event follows a downward trend, indicating the negative signaling to the market.

Keywords: Stock Returns; Tatings; Distribution of Earnings; Inplits; Splits.

\section{RESUMEN}

El objetivo de este trabajo es comprobar el impacto que los eventos corporativos causan no retorno de las acciones de las empresas brasileñas, principalmente los relativos a la divulgación de: a) grupamentos de acciones; B) desdobramento de acciones; C) distribución de dividendos (dividendos y JCP); E d) el cambio de calificaciones de crédito. Diferente los estudios anteriores desarrollados no Brasil sobre este tema, o los estudios de caso de estudios actualizados en Bootstrap. (Com 274 observaciones), grupamentos (con 479 observaciones) y desdobramentos (con 306 observaciones), em número de un número de Eventos de divulgación de dividendos y JCP (com 10.258 observaciones). A partir de una base de datos de 11.317 eventos, los resultados indicaron que las distribuciones de dividendos y JCP influenciaron el mercado positivamente, ya que el retorno de las acciones de $0,70 \%$ no dia do evento. Una divulgación de desdobramentos de las acciones también fue capaz de influir en el mercado, sin que el cambio se produjera en un $1,96 \%$. Por su parte, los grupamentos de acciones no presentados por retornos y ahora estatisticamente significativos, pueden ser inferidos gratificantemente por el retorno de las acciones en este evento.

Palabras-clave: Retorno de Acciones; Nota de Credito; Distribution de Proventos, Grupamentos de Acciones; Desdobramentos de Acciones. 


\section{INTRODUÇÃO}

Os preços das ações negociadas em bolsa de valores são normalmente influenciados por uma ampla gama de fatores, dentre eles alguns eventos econômicos e corporativos, tais como os anúncios de lucros, fusões e aquisições empresariais e de variáveis macroeconômicas. Segundo Mackinlay (1997), os economistas são frequentemente requeridos para medir os efeitos de um evento econômico sobre o valor das firmas, o que parece ser uma tarefa difícil, mas que pode ser atingida com a construção de estudos de eventos.

Os ratings, nesse contexto, foram objetos de estudo de diversas pesquisas. No Brasil, destacam-se os trabalhos de Valle (2002), Damasceno et al. (2008), Freitas e Minardi (2013) e Tonin e Colauto (2015). No exterior, tem-se os estudos de Hsueh e Liu (1992), Vassalou e Xing (2004), Kisgen (2006), Frost (2007), Stolper (2009) e Bolton et al. (2012).

No mercado acionário, o risco é um aspecto importante a ser considerado nas avaliações empresariais e, diante das crises financeiras globais, os ratings divulgados pelas agências de classificação de riscos são fundamentais para o gerenciamento de risco nas empresas. Assim, é imperativo conhecer a dinâmica que envolve a atribuição de ratings às companhias e como o mercado reage diante destas classificações. Ademais, para Freitas e Minardi (2013), os investidores e instituições financeiras confiam nos ratings de crédito porque eles resumem o crédito de um emissor, servindo de importante parâmetro para as decisões de investimento.

Particularmente no mercado de ações, estudos preliminares procuraram analisar o comportamento do preço das ações diante de determinados eventos econômicos, tais como alterações de ratings de crédito divulgados por agências de classificação de risco. Como exemplo, pode-se citar o estudo desenvolvido por Freitas e Minardi (2013), em que o desenvolvimento dos testes estatísticos foi amparado na distribuição $t$ (testes paramétricos); em contrapartida, o que se propõem na presente pesquisa é a adoção de ferramentas estatísticas que não estabeleçam uma distribuição estatística prévia.

Outro evento que pode influenciar nos preços das ações é a divulgação de distribuição de proventos. Com relação à importância dos dividendos e o possível impacto nos preços das ações, Martins e Fama (2012) destacaram que a política de dividendos é um dos assuntos mais controversos no campo das finanças corporativas. Outros estudos, por sua vez, analisaram a reação de mercado aos grupamentos de ações, como os desenvolvidos por Lamoureux e Poon (1987), Hardin III et al. (1997) e Xiao-Xuan (2003). Portanto, dada a sua reconhecida importância, os impactos das divulgações de grupamentos e desdobramentos de ações também serão analisados no presente estudo.

Nesse mesmo contexto, segundo Kothari e Warner (2005), os estudos de eventos examinam o comportamento dos preços das ações das empresas em torno de eventos corporativos. Kramer (2001), entretanto, afirma que o método de inferência estatística dos estudos de eventos baseado em Bootstrap apresenta um melhor desempenho em relação aos métodos tradicionais. Diante desse contexto, surge a seguinte pergunta de pesquisa: como os investidores do mercado acionário percebem as divulgações de grupamentos e desdobramento de ações, de distribuição de proventos e alterações de ratings realizados pelas empresas brasileiras de capital aberto?

Portanto, o objetivo central desta pesquisa é verificar qual o impacto que as divulgações de grupamentos e desdobramento de ações, de distribuição de proventos (dividendos e Juros sobre Capital Próprio - JCP) e das alterações de ratings causam no retorno médio das ações das empresas brasileiras. A inovação desta pesquisa está relacionada à realização de um estudo de eventos com a utilização de método baseado em Bootstrap, abordado por Davinson et al. (1986) e que é livre de qualquer suposição de distribuição específica, ao contrário dos estudos pregressos acima comentados. 
O método Bootstrap foi apresentado inicialmente por Efron (1979) como um processo robusto para estimar a distribuição de dados independentes e identicamente distribuídos. Este procedimento, segundo Kramer (2001), apresenta um melhor desempenho em relação aos métodos tradicionais. Ainda segundo Kramer (2001), o Bootstrap concentra métodos de simulação para avaliar as propriedades de amostragem de estimativas estatísticas, de forma que a presente pesquisa se justifica no ponto em que é necessário compreender a dinâmica que envolve a divulgação de eventos e como o mercado acionário absorve e reage a partir do anúncio destas informações.

Este estudo está divido em cinco partes e é composto por esta introdução, seguido pela revisão dos estudos relevantes, a descrição dos aspectos metodológicos, a análise dos resultados e as considerações finais.

\section{REVISÃO DA LITERATURA}

No ambiente corporativo, Kothari e Warner (2005) afirmam que a magnitude do desempenho anormal dos preços das ações no momento de um evento fornece uma medida de impacto (inesperado) deste evento na riqueza dos detentores de direitos sobre as empresas. Desta forma, ainda segundo Kothari e Warner (2005), os estudos de eventos fornecem elementos relevantes para a compreensão de decisões de políticas corporativas e os reflexos destas políticas no mercado de ações. Nesta mesma linha, Mian e Sankaraguruswamy (2012) apontam que o preço das ações reage aos lucros com componentes racionais e irracionais, o que traz implicações à literatura associada aos estudos de eventos.

Por sua vez, segundo Litzenberger e Ramaswamy (1982), o efeito do pagamento de dividendos nos retornos das ações tem sido um ponto controverso na literatura dessa área. Novis Neto e Saito (2003), inclusive, verificaram o impacto refletido no preço das ações após a divulgação de dividendos - o estudo foi composto de um total de 163 anúncios de pagamentos de dividendos (eventos), foi realizado entre os anos de 1998 e 2000 e incluiu, em sua amostra, as ações mais negociadas na Bolsa de Valores de São Paulo (BM\&FBovespa - $[\mathrm{B}]^{3}$ ) naquele período.

Neste estudo, Novis Neto e Saito (2003) segregaram as amostras em três grupos, cujo critério de separação foi o Dividend Yeld - DY (DY mais baixos, DY intermediários e DY mais altos). Os resultados evidenciaram que as empresas que pagaram maiores dividendos obtiveram um retorno acumulado de $21,97 \%$ nos 90 dias subsequentes ao evento, enquanto que as empresas que pagaram os menores dividendos obtiveram um retorno de negativo de $15,50 \%$.

Novis Neto e Saito (2003) destacaram ainda que as investigações futuras poderiam ser realizadas por um período mais longo e com uma amostra maior. Com base nisso, o presente estudo cumpre estes dois requisitos, ao analisar o efeito do anúncio dos dividendos em um período compreendido entre janeiro de 2005 a março de 2016. Além disso, a amostra foi expandida, de forma que foram analisadas todas as empresas (financeiras e não financeiras) que tiveram anúncios de distribuição de dividendos na BM\&FBovespa (atual $[\mathrm{B}]^{3}$ ) no período considerado, diferentemente de Novis Neto e Saito (2003), que investigaram apenas as ações mais negociadas.

Procianoy e Verdi (2009) analisaram o efeito clientela e a hipótese de sinalização de mercado em pesquisa desenvolvida entre os anos de 1996 até 2000, no mercado acionário brasileiro. Este estudo usou como uma das metodologias o estudo de eventos, tendo os autores reportado que houve um retorno anormal positivo igual a 1,5\% no primeiro dia ex-dividendo, com um nível de significância estatística de $1 \%$. Além disso, os resultados do estudo apontaram que o retorno anormal no dia do evento é positivo e estatisticamente significante para os dividendos inesperados e que os resultados corroboram testes realizados sobre a hipótese de clientela, que encontram um retorno anormal no primeiro dia ex-dividendo. 
O estudo de Procianoy e Verdi (2009) é uma continuação do estudo dos mesmos autores desenvolvido em 2003 (PROCIANOY; VERDI, 2003). O estudo de 2003 foi realizado entre os anos de 1989 até 1993 enquanto que o de estudo de 2009 é uma análise dos anos de 1996 até 2000. Os resultados de ambos os estudos são semelhantes.

Procianoy e Verdi (2003) destacaram ainda a existência de um comportamento irracional do mercado, ao passo em que os investidores estão pagando mais por ações com dividendos do que sem dividendos. Ou seja, o preço da ação no primeiro dia ex-dividendo foi maior do que na data em que o detentor da ação teria direito aos dividendos. Já nos resultados do estudo de Procianoy e Verdi (2009), os autores destacaram que os seus resultados contrariaram o efeito clientela, no entanto confirmaram a teoria da sinalização (em função dos retornos anormais próximos às datas de pagamentos de dividendos).

Nesse contexto, saliente-se que o estudo de Procianoy e Verdi (2009) analisou, em sua amostra, 394 distribuições de dividendos, enquanto que o presente estudo analisa uma amostra que contém, além da distribuição de dividendos, a distribuição de JCP e é composta por um total de 16.087 distribuições compreendidas entre o período de janeiro de 2005 a março de 2016. Além disso, outra diferença fundamental entre os estudos é a adoção da metodologia de Bootstrap, descrita no tópico a seguir, enquanto que o estudo de Procianoy e Verdi (2009) utilizou o teste paramétrico baseado na distribuição $t$.

Martins e Fama (2012), ademais, realizaram uma revisão da literatura, entre os anos de 1990 e 2010, sobre políticas de dividendos no Brasil e destacaram que o estudo sobre o tema dividendos é ainda mais polêmico no Brasil em função de algumas particularidades existentes, destacando ainda a necessidade de estudar-se a realidade brasileira dos JCP e seu tratamento tributário diferenciado. Na pesquisa aqui desenvolvida, a distribuição dos JCP terá tratamento semelhante à distribuição de dividendos, pelas características que eles apresentam.

Para Ross et al. (2015), a política de dividendos pode transmitir informações ao acionista sobre o desempenho da empresa, enquanto Silva et al. (2016) apontam que os dividendos são vistos como sinalizadores de informações ao mercado, com base na Teoria da Sinalização dos Dividendos, tendo neste estudo confirmado a utilidade do dividendo adicional proposto (DAP) como um mecanismo de transmissão de oportunidades e de crescimento futuro dos valores das empresas. O estudo de Silva et al. (2016) foi desenvolvido nos anos de 2010, 2011 e 2012 e analisou o efeito do dividendo adicional proposto (DAP) no preço das ações no mercado de capitais brasileiro.

Nesse sentido, os resultados do estudo de Silva et al. (2016) apontaram que o DAP é uma informação relevante ao mercado de capitais. Outro estudo desenvolvido no Brasil foi realizado por Freire et al. (2005) e investigou a relação entre o dividendo yeld e o lucro anormal, concluindo que esta relação não existe para o período analisado, compreendido entre os anos de 1996 a 2001.

Fama et al. (1969) examinaram se os desdobramentos de ações (Splits) são associados com retorno não usuais de ações e destacaram que os últimos desdobramentos verificados têm sido muitas vezes associados a aumentos substanciais de dividendos. Outro estudo que analisou os desdobramentos foi o desenvolvido por Lamoureux e Poon (1987), que estabeleceu que a partir dos Splits há um aumento no número de transações juntamente com o número de ações negociadas o que aumenta a volatilidade da série de preços das ações.

Além disso, Lamoureux e Poon (1987) estabelecem que há uma reação anormal positiva aos anúncios de desdobramentos. Já o estudo de Xiao-Xuan (2003) investigou a reação do mercado aos Splits no mercado chinês entre os anos de 2007 e 2010 e encontrou significantes retornos anormais em torno da data do anúncio e, em especial, antes da data do anúncio. Por sua vez, o grupamento de ações foi analisado no Brasil por Comiran (2009), que em estudo desenvolvido entre os anos de 1986 a 2008, concluiu que o grupamento de ações não possui qualquer efeito sobre os preços das ações. 
Com relação às alterações de ratings, Hsueh e Liu (1992) concluíram que os anúncios de mudanças de ratings trouxeram mais informações quando houve mais incerteza no mercado e o impacto do anuncio da mudança (tanto em downgrades e upgrades) é realmente mais forte nas empresas com menos informações disponíveis, o que reafirma que a informação é relevante (ao ponto de afetar o preço da ação) se ela é nova e, consequentemente, valiosa aos investidores.

A pesquisa de Hsueh e Liu (1992) compreendeu o período de maio de 1982 até julho de 1987 e a amostra foi composta por 761 dívidas corporativas emitidas por empresas listadas na NYSE e AMEX com ratings anunciados pela Moody's.

Por fim, segundo Kisgen (2006), os ratings de crédito impactam nas decisões de estrutura de capital por parte dos gestores enquanto que para Stolper (2009), muitas vezes, as agências de classificação de risco têm incentivos para atribuir classificações inflacionadas. Desta forma, justifica-se a inclusão desses eventos (divulgação de ratings de crédito) no estudo em questão.

\section{MÉTODO DE PESQUISA E BASE DE DADOS}

\subsection{Retornos anormais}

A metodologia empregada nesta pesquisa está alinhada com os estudos desenvolvidos por Moshirian et al. (2009) e Freitas e Minardi (2013). Para tanto, os retornos das ações foram calculados nos termos da equação (1) a seguir:

$$
R_{i, t}=\left[\left(\frac{P_{i, t}}{P_{i, t-1}}\right)-1\right] \times 100
$$

Onde:

$R_{i, t}=$ retorno da ação i no tempo t;

$P_{i, t}=$ preço de fechamento da ação i no tempo t;

$P_{i, t-1}=$ preço da ação i no trimestre $\mathrm{t}-1$.

Após o cálculo dos retornos de cada ação individualmente, foram calculados os retornos anormais (AR) das ações de acordo com a equação (2), apresentada a seguir:

$$
A R_{i, \tau}=\prod_{t=1}^{\tau}\left(1+R_{i, t}\right)-\prod_{t=1}^{\tau}\left(1+\operatorname{RIbov}_{t}\right)
$$

Onde:

$A R_{i, \tau}=$ retorno anormal da ação i no tempo $\tau$;

$R_{i, t}=$ retorno da ação i no tempo t;

$\operatorname{RIbov}_{t}=$ retorno da carteira de mercado (Ibovespa) no tempo $\mathrm{t}$.

Vale destacar que a adoção do índice Ibovespa como proxy para o comportamento de mercado está alinhada com os estudos de Moshirian et al. (2009), Freitas e Minardi (2013) e Silva et al. (2016). Ressaltamos que o modelo de retorno ajustado ao mercado é uma simplificação do modelo de mercado.

Em seguida foram estimados os retornos anormais acumulados (CAR) e os retornos anormais acumulados médios ( $\overline{\mathrm{CAR}})$, por meio das equações (3) e (4), como seguem: 


$$
\begin{gathered}
C A R=\sum_{t=0}^{\tau} A R_{i, \tau} \\
\overline{C A R}=\frac{\sum_{t=0}^{\tau} A R_{i, \tau}}{N}
\end{gathered}
$$

Onde:

$C A R=$ retornos anormais acumulados da ação i no tempo $\tau$;

$A R_{i, \tau}=$ retorno anormal da ação i no tempo $\tau$;

$N=$ número de ações incluídas na média.

\subsection{Estudos de eventos}

Os estudos de eventos foram apresentados originalmente por Ball e Brown (1968) e Fama et al. (1969) e, ao longo das últimas quatro décadas, forneceram uma enorme contribuição para a pesquisa em mercado de capitais, como aponta Corrado (2011), sendo usualmente utilizados para verificar o impacto, no preço das ações, de eventos corporativos. Mackinlay (1997), ressalta que os economistas são frequentemente solicitados para medir os impactos de um evento econômico sobre o valor das firmas, objetivo que pode ser atingido com a construção de estudos de eventos.

Tal ferramenta foi inicialmente desenvolvida para aplicação em finanças e contabilidade, de forma que, para Kothari e Warner (2005), os estudos de eventos examinam o comportamento dos preços das ações das empresas em torno de eventos corporativos e, além disso, servem a um propósito importante na investigação do mercado de capitais, como forma de testes de eficiência do mercado.

Segundo Corrado (2011), os estudos de eventos foram desenvolvidos como uma ferramenta estatística para pesquisas empíricas em contabilidade e finanças. No entanto, esta ferramenta tem migrado para outras disciplinas como economia, história, direito, administração, marketing e ciências políticas.

A utilização de estudos de eventos baseado em Bootstrap é o que diferencia este estudo dos anteriores desenvolvidos no Brasil, consoante discutido no primeiro item deste estudo. Além disso, o uso de estudo de eventos está alinhado com o que fora destacado por Mackinlay (1997) e Kothari e Warner (2005), ou seja, pretende-se avaliar o efeito de eventos corporativos de a) grupamentos de ações; b) desdobramento de ações; c) distribuição de proventos (dividendos e JCP); e d) alterações de ratings de crédito nos preços das ações das empresas brasileiras de capital aberto listadas na $[\mathrm{B}]^{3}$.

O principal benefício da utilização deste método é o ajuste para pequenas amostras, como é o caso dos eventos coletados de alterações de ratings (com 274 observações), grupamentos (com 479 observações) e desdobramentos (com 306 observações), em comparação ao número de eventos de divulgações de dividendos e JCP (com 10.258 observações).

Outro ponto que merece ser destacado é a simulação realizada nas amostras para o ajuste à melhor distribuição estatística, o que traz maior robustez para os resultados encontrados.

Chou (2004) destaca que testes estatísticos para estudos de eventos multivariados são baseados na normalidade multivariada e que há evidências prévias que indicam que as performances destes testes não são satisfatórias, em especial porque os retornos estão longe de serem distribuídos normalmente (em especial quando se trata de retornos diários). Posto isso, Chou (2004) ressalta, ainda, que o uso de métodos baseados em Bootstrap são livres de qualquer suposição de distribuição específica e fornecem melhores aproximações às distribuições de amostragem de estatísticas de teste em estudos de eventos multivariados. 
Vale ressaltar que o estudo desenvolvido por Chou (2004) foi realizado na bolsa de valores de Tóquio (Tókio Stock Exchange) e que os resultados estão alinhados ao que fora apontado por Kramer (2001), ou seja, o método de inferência estatística dos estudos de eventos baseado em Bootstrap apresenta um melhor desempenho em relação aos métodos tradicionais.

Os estudos de Kramer (2001) e de Chou (2004) apresentaram a superioridade de uso de métodos baseados em Bootstrap e servem como embasamento da escolha metodológica adotada nesta pesquisa.

Os testes do presente estudo foram realizados por meio do Software $R$ com a utilização do pacote estatístico eventstudies, desenvolvido por Shah et al. (2013), cuja abordagem específica utilizada no pacote é a de Davinson et al. (1986). Além disso, o Bootstrap gera um intervalo de confiança de $2,5 \%$ e $97,5 \%$ para as estimativas calculadas, o que reforça a robustez da metodologia eleita para as análises do presente estudo.

\subsection{Definição da janela de evento}

Neste estudo foi considerada a janela de evento de 15 dias antes e depois dos eventos para poder abranger o período considerado por Sena et al. (2016) que fora de 12 dias antes e depois. Para Camargos e Barbosa (2003) a escolha da janela do evento envolve certo grau de arbitrariedade em função do evento a ser estudado e não deve ser muito longa pois poderia haver o reflexo de outros eventos distintos aos estudados.

\subsection{Base de dados}

Os eventos corporativos que compõem a base de dados analisada nesta pesquisa são caracterizados por: a) divulgações de grupamentos de ações; b) divulgações de desdobramento de ações; c) divulgações de distribuição de proventos (dividendos e JCP); e d) das alterações de ratings de crédito. Os períodos considerados em cada tipo de evento, assim como o número de eventos analisados, estão apresentados na Tabela 1. Procurou-se analisar, pelo menos, de janeiro de 2005 até dezembro de 2015. No entanto, alguns eventos tiveram como início de análise a primeira ocorrência na bolsa de valores.

Vale destacar que as alterações de ratings foram extraídas da base de dados Thomson One Analytics ${ }^{\circledR}$ enquanto que as demais variáveis foram extraídas da base de dados Economática ${ }^{\circledR}$. Além disso, é fundamental destacar que a amostra é composta por empresas financeiras e não financeiras de capital aberto listadas na $[\mathrm{B}]^{3}$ que apresentaram algum dos eventos estudados.

E é imperativo ressalvar que um ponto forte deste estudo está na amplitude dos períodos analisados: para os desdobramentos e grupamentos considerou-se desde a primeira ocorrência na bolsa de valores brasileira até a última ocorrência até janeiro de 2016; para as alterações de ratings foram analisados 16 anos; e, para as distribuições de proventos, foram analisados 11 anos.

Tabela 1 - Sumário dos eventos considerados na pesquisa

\begin{tabular}{lccc}
\hline Variáveis/Eventos & Número de observações & Período analisado & Evento inicial e final \\
\hline Alterações de ratings & 274 & jan/1999 - dez/2015 & $15 / 01 / 1999$ e 08/12/2015 \\
Desdobramentos & 306 & nov/1992 - jan/2016 & 26/11/1992 e 14/01/2016 \\
Grupamentos & 479 & ago/1989-jan/2016 & 24/08/1989 e 26/01/2016 \\
Proventos (dividendos e JCP) & 10.258 & jan/2005 - mar/2016 & 03/01/2005 e 11/03/2016 \\
\hline
\end{tabular}

\section{ANÁLISE DE RESULTADOS}

A análise inicial é desenvolvida a partir do comportamento das ações em torno dos Desdobramentos e Grupamentos que estão apresentados na Tabela 2. Nela, pode-se verificar 
que as ações apresentam retornos anormais médios positivos após os desdobramentos. No dia do evento, o retorno médio anormal foi de $1,96 \%$ e os retornos positivos persistiram nos dias subsequentes. Sobretudo, pode-se ressaltar, com um nível de significância de $95 \%$, que o retorno anormal no dia do desdobramento é positivo e este ponto pode ser verificado nos limites interiores $(0,35 \%)$ e superior de $(3,89 \%)$.

Já os Grupamentos refletiram negativamente nos retornos das ações com um retorno anormal médio negativo de $1,50 \%$ e com um limite inferior também negativo de 2,98\%, como pode ser verificado na Tabela 2.

Tabela 2 - Retorno anormal acumulado em torno dos desdobramentos e grupamentos

\begin{tabular}{|c|c|c|c|c|c|c|}
\hline \multirow[t]{2}{*}{$\begin{array}{l}\text { Dias em torno do } \\
\text { evento }\end{array}$} & \multicolumn{3}{|c|}{$\begin{array}{c}\text { Desdobramentos (Retorno \%) } \\
\text { Intervalo de confiança }\end{array}$} & \multicolumn{3}{|c|}{$\begin{array}{c}\text { Grupamentos (Retorno \%) } \\
\text { Intervalo de confiança }\end{array}$} \\
\hline & $2,50 \%$ & Média & $97,50 \%$ & $2,50 \%$ & Média & $\mathbf{9 7 , 5 0 \%}$ \\
\hline-15 & $-0,497$ & $-0,234$ & 0,031 & $-0,709$ & $-0,253$ & 0,198 \\
\hline-14 & $-0,761$ & 0,003 & 1,091 & $-0,839$ & $-0,347$ & 0,180 \\
\hline-13 & $-0,901$ & 0,023 & 1,220 & $-0,910$ & $-0,246$ & 0,417 \\
\hline-12 & $-0,811$ & 0,160 & 1,442 & $-0,948$ & $-0,278$ & 0,369 \\
\hline-11 & $-0,804$ & 0,197 & 1,441 & $-1,113$ & $-0,253$ & 0,603 \\
\hline-10 & $-0,777$ & 0,096 & 1,181 & $-1,212$ & $-0,281$ & 0,732 \\
\hline-9 & $-0,579$ & 0,344 & 1,395 & $-1,096$ & $-0,187$ & 0,820 \\
\hline-8 & $-0,075$ & 1,035 & 2,460 & $-1,684$ & $-0,764$ & 0,202 \\
\hline-7 & 0,305 & 1,668 & 3,271 & $-2,024$ & $-0,946$ & 0,096 \\
\hline-6 & 0,696 & 2,094 & 3,874 & $-1,900$ & $-0,846$ & 0,221 \\
\hline-5 & 0,825 & 2,155 & 3,889 & $-2,102$ & $-0,867$ & 0,324 \\
\hline-4 & 0,549 & 1,835 & 3,497 & $-2,077$ & $-0,835$ & 0,530 \\
\hline-3 & 0,377 & 1,735 & 3,498 & $-2,075$ & $-0,751$ & 0,620 \\
\hline-2 & 0,229 & 1,663 & 3,509 & $-2,322$ & $-1,048$ & 0,349 \\
\hline-1 & 0,049 & 1,533 & 3,405 & $-2,629$ & $-1,286$ & 0,166 \\
\hline 0 & 0,355 & 1,961 & 3,886 & $-2,977$ & $-1,497$ & 0,074 \\
\hline 1 & 0,551 & 2,348 & 4,477 & $-3,587$ & $-2,093$ & $-0,493$ \\
\hline 2 & 0,847 & 2,578 & 4,701 & $-3,708$ & $-2,126$ & $-0,379$ \\
\hline 3 & 0,990 & 2,755 & 4,867 & $-3,804$ & $-2,205$ & $-0,411$ \\
\hline 4 & 1,111 & 2,856 & 4,956 & $-3,943$ & $-2,208$ & $-0,371$ \\
\hline 5 & 0,934 & 2,557 & 4,439 & $-3,959$ & $-2,168$ & $-0,296$ \\
\hline 6 & 0,796 & 2,493 & 4,548 & $-3,682$ & $-1,869$ & 0,057 \\
\hline 7 & 0,923 & 2,670 & 4,794 & $-3,529$ & $-1,574$ & 0,536 \\
\hline 8 & 0,595 & 2,286 & 4,342 & $-3,996$ & $-1,968$ & 0,224 \\
\hline 9 & 0,584 & 2,460 & 4,626 & $-4,197$ & $-2,202$ & $-0,047$ \\
\hline 10 & 0,200 & 2,013 & 4,226 & $-4,826$ & $-2,722$ & $-0,645$ \\
\hline 11 & $-0,023$ & 1,917 & 4,185 & $-5,536$ & $-3,322$ & $-1,252$ \\
\hline 12 & $-0,073$ & 1,848 & 4,190 & $-5,614$ & $-3,440$ & $-1,303$ \\
\hline 13 & $-0,067$ & 2,091 & 4,526 & $-5,879$ & $-3,703$ & $-1,522$ \\
\hline 14 & $-0,088$ & 2,136 & 4,727 & $-6,311$ & $-4,035$ & $-1,874$ \\
\hline 15 & $-0,047$ & 2,153 & 4,829 & $-6,368$ & $-3,998$ & $-1,768$ \\
\hline
\end{tabular}

Em seguida, nos Figuras 1 e 2 é possível analisar os comportamentos graficamente com a evidência de que as ações apresentaram um retorno anormal médio positivo após os desdobramentos, enquanto que os grupamentos foram seguidos de retornos anormais médios negativos. 
Para Ross et al. (2015), uma empresa, ao anunciar um desdobramento, aumenta o número de ações existentes e com isso cada ação passa a ter direito a um valor menor de fluxo de caixa e o preço das ações deveria cair. No presente estudo, compreendido entre novembro/1992 e janeiro/2016, não foi o que ocorreu de fato, pois houve um retorno anormal positivo no dia do evento até o décimo dia subsequente, como se pode verificar no Figura 1.

Os resultados encontrados no presente estudo estão alinhados com o que fora encontrado pelos estudos desenvolvidos por Lamoureux e Poon (1987) e Xiao-Xuan (2003), que igualmente encontram retornos anormais significantes e positivos em torno dos anúncios de desdobramentos (splits).

\section{Figura 1 - Comportamento das ações em torno dos desdobramentos}

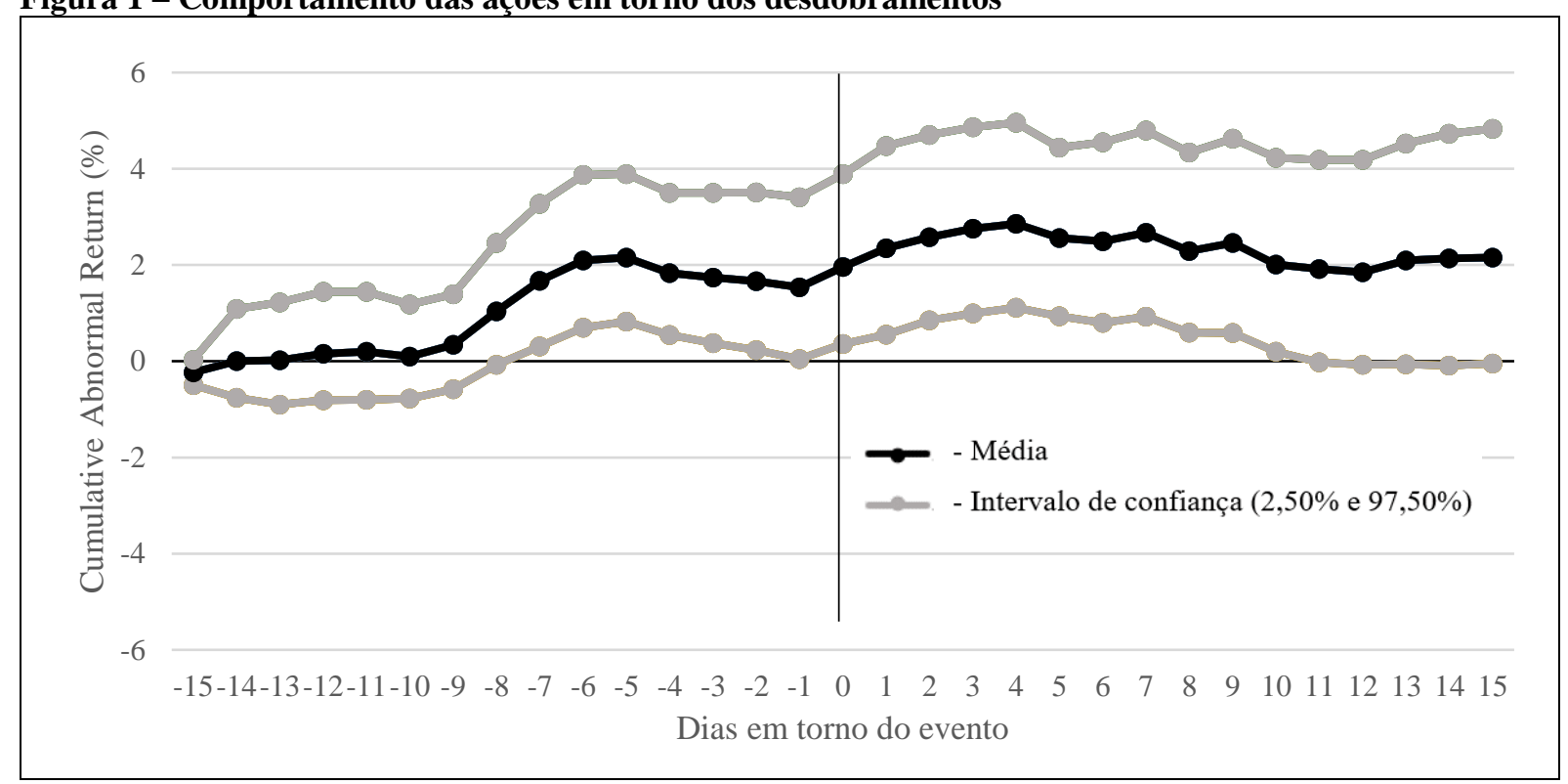

Figura 2 - Comportamento das ações em torno dos grupamentos

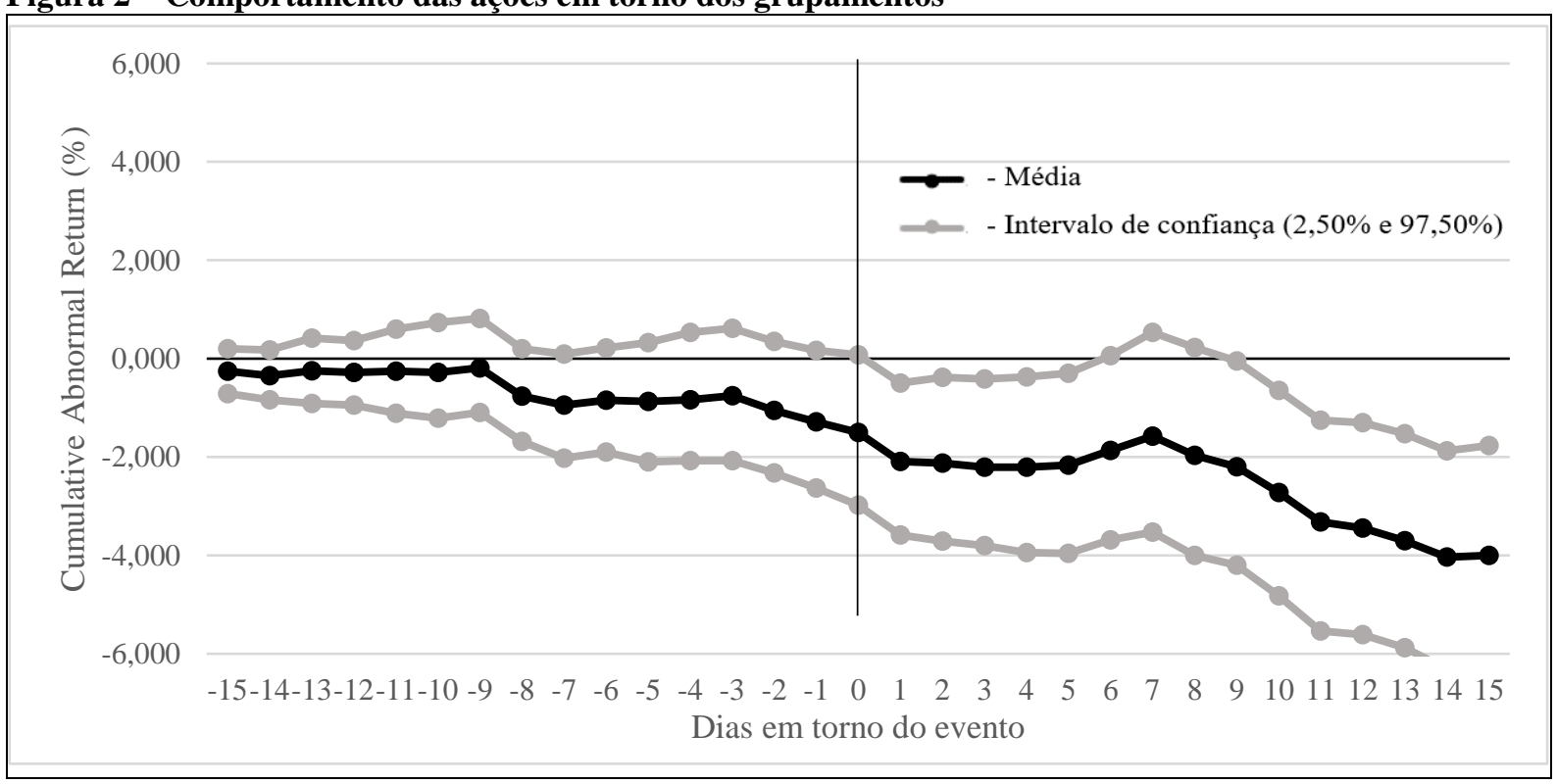

O que diferencia os estudos de Lamoureux e Poon (1987) e Xiao-Xuan (2003) ao aqui desenvolvido é a utilização a distribuição paramétrica $t$ de Student. Além disso, pode-se perceber que os retornos anormais apresentados no mercado chinês, reportados por Xiao-Xuan 
(2003), são substancialmente maiores do que os apresentados aqui $(\overline{C A R})$ próximo de $4 \%$ no dia do evento), fato que é explicado pela adoção do Bootstrap.

Ademais, para analisar o comportamento do mercado às alterações de ratings empresariais foram separadas as alterações positivas (aumentos das notas - upgrades) e os rebaixamentos das notas (downgrades) e testado o comportamento do mercado de ações à estas alterações.

Estão apresentadas na Tabela 3 as reações do mercado aos upgrades e downgrades e pode-se verificar que o retorno anormal acumulado das ações das empresas que tiveram suas notas rebaixadas é positivo no dia do evento $(0,20 \%)$ e no dia subsequente $(0,52 \%)$.

Tabela 3 - Retorno anormal acumulado em torno dos Upgrades e Downgrades de ratings

\begin{tabular}{|c|c|c|c|c|c|c|}
\hline \multirow{2}{*}{$\begin{array}{c}\text { Dias em } \\
\text { torno do } \\
\text { evento }\end{array}$} & \multicolumn{3}{|c|}{$\begin{array}{c}\text { Upgrades } \\
\text { Intervalo de confiança }\end{array}$} & \multicolumn{3}{|c|}{$\begin{array}{c}\text { Downgrades } \\
\text { Intervalo de confiança }\end{array}$} \\
\hline & $2,50 \%$ & Média & $97,50 \%$ & $2,50 \%$ & Média & $\mathbf{9 7 , 5 0 \%}$ \\
\hline-15 & $-0,636$ & $-0,217$ & 0,182 & $-0,080$ & 0,239 & 0,603 \\
\hline-14 & $-0,693$ & $-0,103$ & 0,531 & $-0,199$ & 0,248 & 0,724 \\
\hline-13 & $-1,282$ & $-0,519$ & 0,202 & $-0,064$ & 0,485 & 1,137 \\
\hline-12 & $-1,148$ & $-0,118$ & 0,844 & 0,017 & 0,568 & 1,199 \\
\hline-11 & $-1,678$ & $-0,257$ & 1,029 & 0,061 & 0,673 & 1,314 \\
\hline-10 & $-1,008$ & 0,344 & 1,630 & 0,256 & 0,879 & 1,510 \\
\hline-9 & $-1,337$ & 0,083 & 1,377 & $-0,092$ & 0,718 & 1,575 \\
\hline-8 & $-1,527$ & $-0,190$ & 1,094 & 0,163 & 0,969 & 1,816 \\
\hline-7 & $-1,714$ & $-0,291$ & 1,008 & $-0,163$ & 0,735 & 1,645 \\
\hline-6 & $-2,118$ & $-0,552$ & 0,945 & $-0,079$ & 0,802 & 1,705 \\
\hline-5 & $-2,369$ & $-0,735$ & 0,791 & $-0,100$ & 0,891 & 1,878 \\
\hline-4 & $-2,516$ & $-0,890$ & 0,716 & $-0,155$ & 0,900 & 1,890 \\
\hline-3 & $-3,068$ & $-1,239$ & 0,559 & $-0,151$ & 0,879 & 1,908 \\
\hline-2 & $-3,152$ & $-1,177$ & 0,719 & 0,092 & 1,147 & 2,260 \\
\hline-1 & $-4,204$ & $-1,930$ & 0,212 & $-0,783$ & 0,548 & 1,847 \\
\hline 0 & $-4,596$ & $-2,166$ & 0,121 & $-1,533$ & 0,198 & 1,587 \\
\hline 1 & $-5,277$ & $-2,631$ & $-0,267$ & $-0,987$ & 0,516 & 1,848 \\
\hline 2 & $-5,228$ & $-2,605$ & $-0,226$ & $-1,921$ & $-0,109$ & 1,385 \\
\hline 3 & $-5,113$ & $-2,485$ & $-0,046$ & $-1,938$ & $-0,119$ & 1,426 \\
\hline 4 & $-5,611$ & $-2,754$ & $-0,227$ & $-2,132$ & $-0,113$ & 1,536 \\
\hline 5 & $-5,283$ & $-2,485$ & 0,089 & $-1,922$ & 0,143 & 1,795 \\
\hline 6 & $-6,059$ & $-2,938$ & $-0,133$ & $-1,935$ & 0,095 & 1,803 \\
\hline 7 & $-6,237$ & $-2,894$ & $-0,012$ & $-1,860$ & 0,148 & 1,800 \\
\hline 8 & $-6,821$ & $-3,080$ & $-0,048$ & $-1,573$ & 0,482 & 2,203 \\
\hline 9 & $-7,126$ & $-3,392$ & $-0,392$ & $-2,044$ & 0,143 & 1,888 \\
\hline 10 & $-5,861$ & $-2,439$ & 0,437 & $-1,743$ & 0,348 & 2,101 \\
\hline 11 & $-6,199$ & $-2,541$ & 0,527 & $-1,767$ & 0,295 & 2,012 \\
\hline 12 & $-6,106$ & $-2,392$ & 0,813 & $-2,473$ & $-0,092$ & 1,852 \\
\hline 13 & $-6,336$ & $-2,508$ & 0,652 & $-2,496$ & $-0,078$ & 2,078 \\
\hline 14 & $-6,655$ & $-2,504$ & 0,822 & $-2,000$ & 0,258 & 2,249 \\
\hline 15 & $-6,237$ & $-2,150$ & 1,243 & $-1,943$ & 0,438 & 2,441 \\
\hline
\end{tabular}

Estes resultados indicam que, em termos médios, os retornos em torno de rebaixamentos de notas são positivos com uma pequena queda nos cinco dias que antecederam ao 
rebaixamento. Já o retorno das ações que tiveram um aumento em sua nota apresentou um retorno anormal médio negativo no dia do evento de $2,2 \%$.

Os Figuras 3 e 4 apresentam o comportamento das ações em torno da divulgação de upgrades e downgrades de ratings, respectivamente, por analistas de mercado.

Comparativamente, no estudo de Freitas e Minardi (2013), foram analisadas 138 alterações de ratings de empresas brasileiras entre os anos de 2000 e 2009. Já o presente estudo analisa 274 alterações. A principal divergência nos resultados entre os dois estudos é com relação aos downgrades de ratings. Enquanto os resultados de Freitas e Minardi (2013) apontaram para um $\overline{C A R}$ negativo de $6,35 \%$ (significante a $5 \%$ ) aqui fora reportado que os rebaixamentos de ratings não apresentaram significância estatística em especial pela utilização do método baseado em Bootstrap.

Figura 3 - Comportamento das ações em torno dos upgrades de ratings

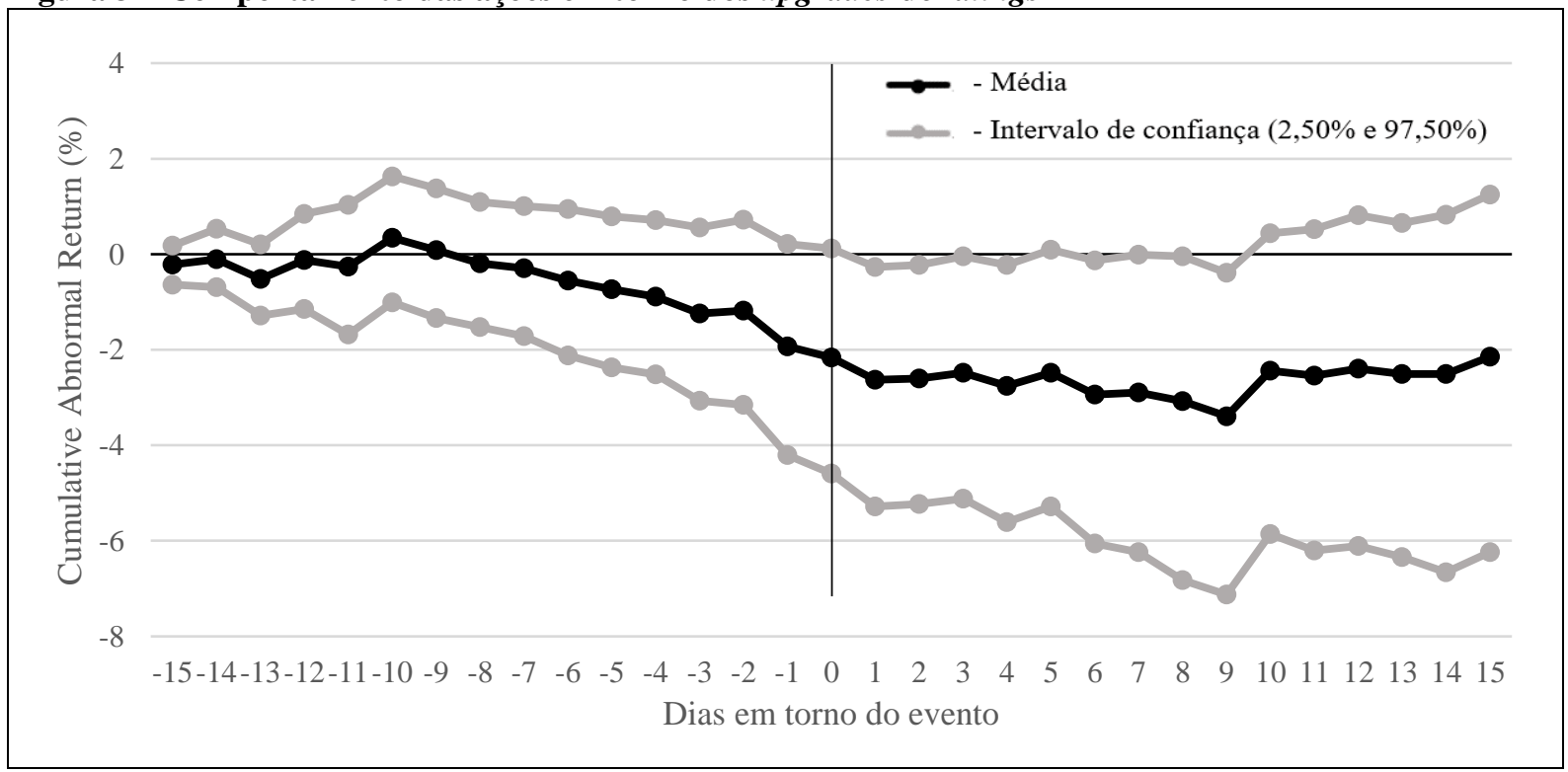

Figura 4 - Comportamento das ações em torno dos downgrades de ratings

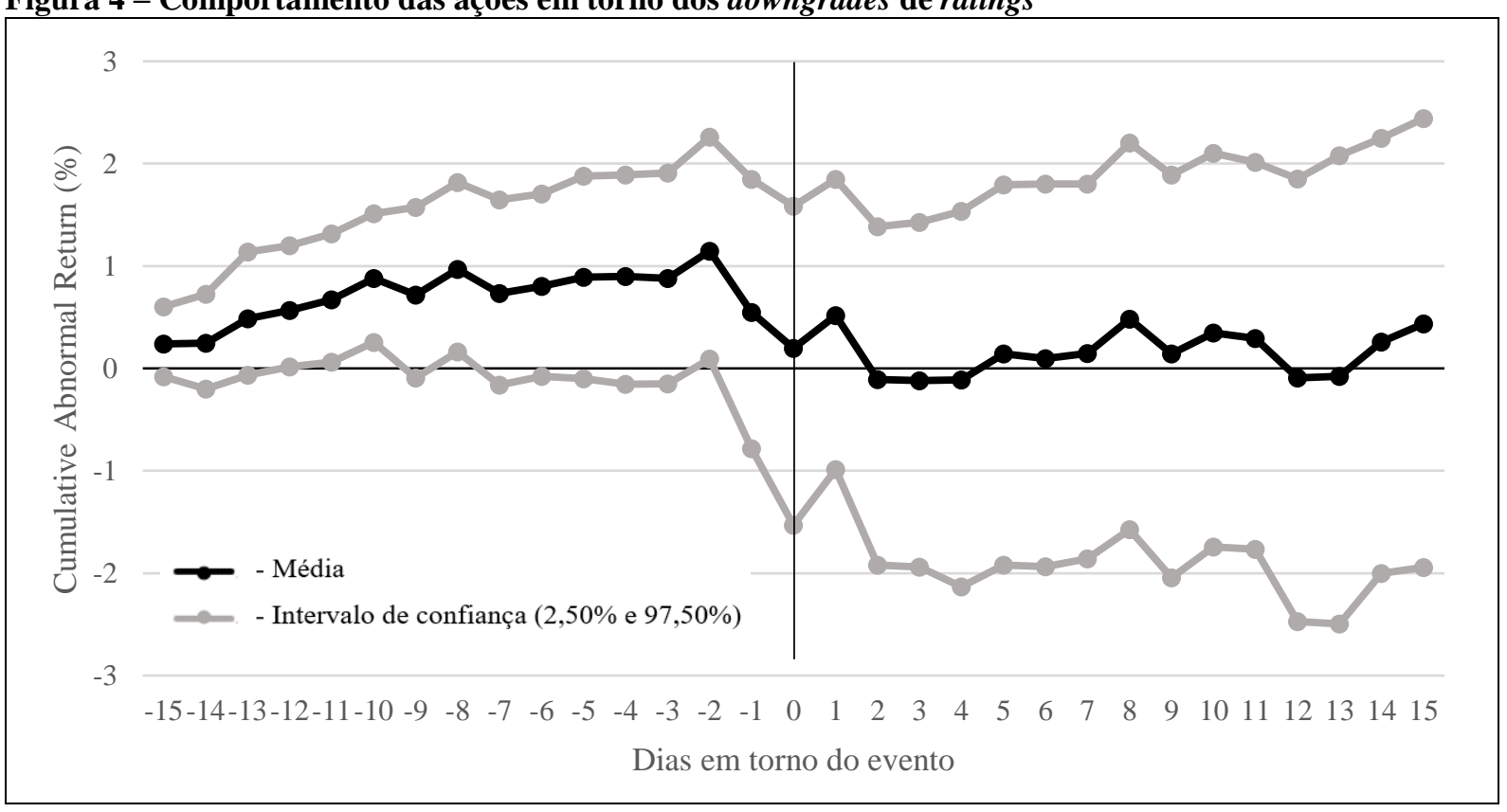


Na sequência, a Tabela 4 apresenta o retorno anormal acumulado, verificado em torno dos eventos de distribuição de dividendos e de JCP, porquanto o Figura 5 apresenta o comportamento das ações em torno de mencionados eventos.

A partir dos resultados reportados pela Tabela 4 pode-se inferir que o retorno acumulado médio $(\overline{C A R})$ das ações, que divulgaram a distribuição de proventos, foi de $0,703 \%$ e que os limites de significância de 2,5\% e 97,5\% também foram positivos. O que indica que a distribuição de dividendos e de JCP influenciaram o mercado positivamente. Vale destacar que o que fora reportado aqui é alinhado com o que fora apresentado anteriormente por Procianoy e Verdi (2009).

Tabela 4 - Retorno anormal acumulado em torno de distribuições de dividendos e JCP

\begin{tabular}{|c|c|c|c|}
\hline \multirow{2}{*}{$\begin{array}{c}\text { Dias em } \\
\text { torno do } \\
\text { evento }\end{array}$} & \multicolumn{3}{|c|}{$\begin{array}{c}\text { Proventos } \\
\text { Intervalo de confiança }\end{array}$} \\
\hline & $2,50 \%$ & Média & $97,50 \%$ \\
\hline-15 & $-0,027$ & 0,019 & 0,067 \\
\hline-14 & $-0,095$ & $-0,034$ & 0,024 \\
\hline-13 & $-0,112$ & $-0,038$ & 0,035 \\
\hline-12 & $-0,057$ & 0,029 & 0,115 \\
\hline-11 & $-0,002$ & 0,095 & 0,189 \\
\hline-10 & 0,063 & 0,159 & 0,255 \\
\hline-9 & 0,154 & 0,269 & 0,375 \\
\hline-8 & 0,179 & 0,292 & 0,404 \\
\hline-7 & 0,218 & 0,335 & 0,447 \\
\hline-6 & 0,200 & 0,322 & 0,450 \\
\hline-5 & 0,318 & 0,447 & 0,567 \\
\hline-4 & 0,353 & 0,492 & 0,624 \\
\hline-3 & 0,419 & 0,555 & 0,696 \\
\hline-2 & 0,395 & 0,541 & 0,682 \\
\hline-1 & 0,488 & 0,642 & 0,796 \\
\hline 0 & 0,544 & 0,703 & 0,863 \\
\hline 1 & 0,778 & 0,950 & 1,123 \\
\hline 2 & 0,731 & 0,909 & 1,092 \\
\hline 3 & 0,777 & 0,959 & 1,145 \\
\hline 4 & 0,880 & 1,079 & 1,279 \\
\hline 5 & 0,853 & 1,050 & 1,248 \\
\hline 6 & 0,821 & 1,036 & 1,241 \\
\hline 7 & 0,873 & 1,084 & 1,286 \\
\hline 8 & 0,906 & 1,127 & 1,324 \\
\hline 9 & 0,996 & 1,219 & 1,427 \\
\hline 10 & 1,036 & 1,269 & 1,484 \\
\hline 11 & 1,083 & 1,321 & 1,539 \\
\hline 12 & 1,146 & 1,386 & 1,619 \\
\hline 13 & 1,268 & 1,514 & 1,759 \\
\hline 14 & 1,318 & 1,569 & 1,813 \\
\hline 15 & 1,302 & 1,544 & 1,781 \\
\hline
\end{tabular}

É imperativo destacar que o resultado de Procianoy e Verdi (2009) foi de que as divulgações dos dividendos levaram à um retorno anormal positivo igual a $1,5 \%$ no primeiro 
dia ex-dividendo enquanto reportamos aqui um $\overline{C A R}$ menor de $0,703 \%$ mas considerando uma amostra e um período maior que àqueles autores.

Além disso, utilizando um método baseado em Bootstrap que é livre de qualquer suposição de distribuição específica e que fornecem melhores aproximações às distribuições de amostragem de estatísticas de teste em estudos de eventos multivariados como apontado por Chou (2004).

Figura 5 - Comportamento das ações em torno de distribuições de dividendos e JCP

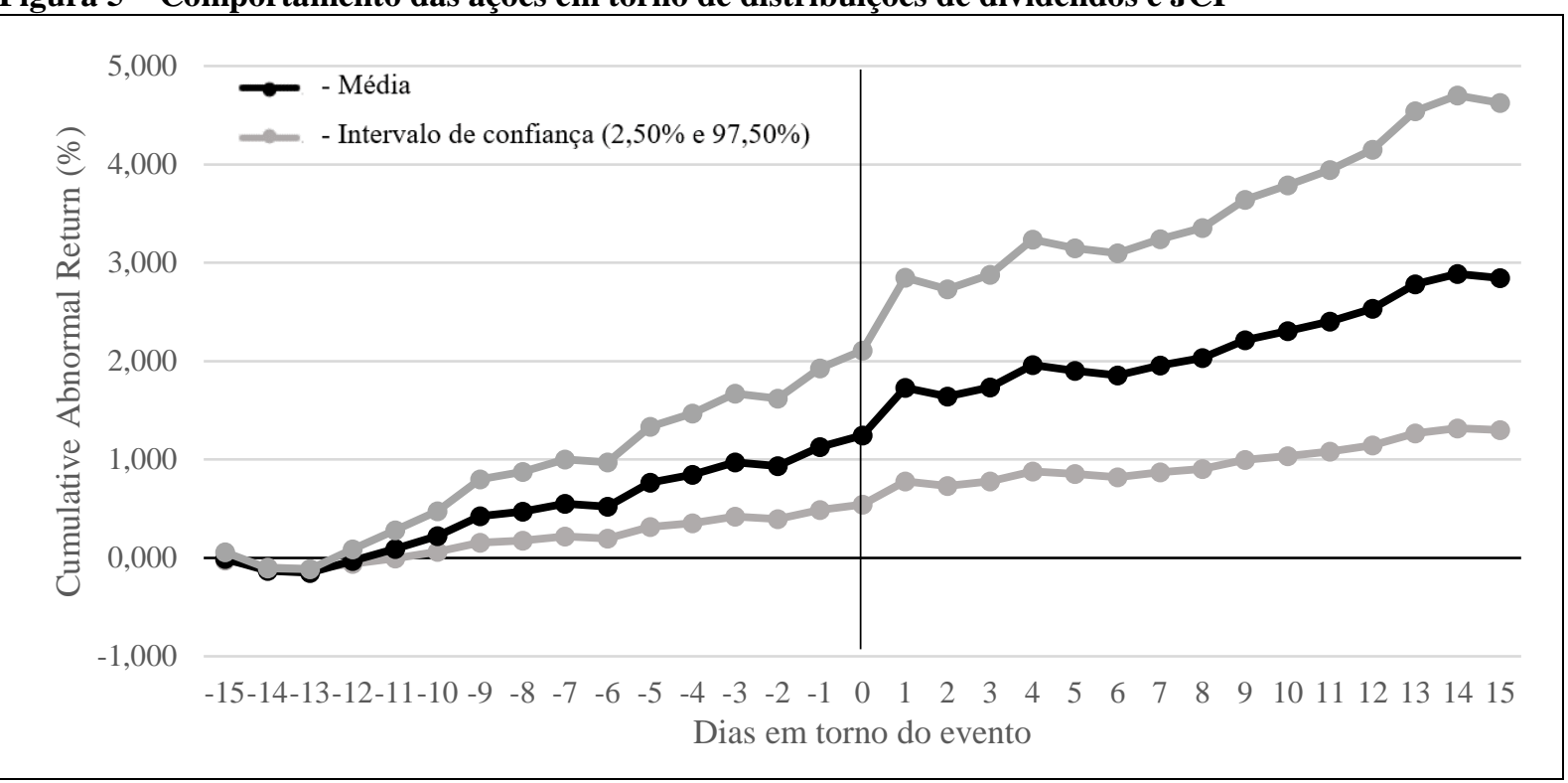

Após a realização dos testes e com o objetivo de responder à pergunta de pesquisa ("como os investidores do mercado acionário percebem as divulgações de grupamentos e desdobramento de ações, de distribuição de proventos e alterações de ratings realizados pelas empresas brasileiras de capital aberto?") verificou-se que, resumidamente, as distribuições de proventos afetaram positivamente o mercado brasileiro entre janeiro de 2005 e março de 2016.

Outro evento que influenciou o mercado acionário foi a divulgação de desdobramentos de ações, uma vez que no dia do evento o retorno médio anormal das ações foi de $1,96 \%$, também considerando um intervalo de confiança de $95 \%$. Com o objetivo de sumarizar os resultados, o Quadro 1 abaixo foi desenvolvido com esse propósito.

Quadro 1 - Sumário dos resultados encontrados

\begin{tabular}{|l|l|}
\hline Eventos & Resultados \\
\hline Grupamentos de ações & $\begin{array}{l}\text { Alinhado às características atreladas aos grupamentos (baixo } \\
\text { valor das ações a serem agrupadas, por exemplo), esse evento } \\
\text { não apresentou retornos anormais estatisticamente } \\
\text { significantes; mas, graficamente, pode-se verificar uma redução } \\
\text { nos preços das ações. }\end{array}$ \\
\hline Desdobramentos de ações & $\begin{array}{l}\text { Influência positiva levando à um aumento nos preços das ações, } \\
\text { com um retorno acumulado anormal de 1,96\%. Resultados } \\
\text { alinhados aos estudos de Lamoureux e Poon (1987) e Xiao- } \\
\text { Xuan (2003) }\end{array}$ \\
\hline Distribuição de proventos (dividendos e JCP) & $\begin{array}{l}\text { Leva à um aumento nos preços das ações, com um retorno } \\
\text { acumulado anormal de 0,70\%. }\end{array}$ \\
\hline Alterações de ratings de crédito & $\begin{array}{l}\text { Não houve retornos anormais estatisticamente significantes. } \\
\text { Resultados divergentes do que fora apresentado por Freitas e } \\
\text { Minardi (2013). }\end{array}$ \\
\hline
\end{tabular}




\section{CONSIDERAÇÕES FINAIS}

Alguns eventos corporativos podem influenciar os preços de mercado das ações, dentre os quais se destacam: a) divulgações de grupamentos de ações; b) divulgações de desdobramento de ações; c) divulgações de distribuição de proventos (dividendos e JCP); e d) alterações de ratings de crédito emitidos por agências de classificação de risco. A proposta deste estudo foi a de verificar se esses eventos corporativos influenciaram o preço das ações, levando a retornos anormais em torno das datas de anúncios destes fatos. Desse modo procurouse responder à pergunta de pesquisa: como os investidores do mercado acionário percebem as divulgações de grupamentos e desdobramento de ações, de distribuição de proventos e alterações de ratings realizados pelas empresas brasileiras de capital aberto?

A partir do que fora exposto, a principal inovação desta pesquisa foi a utilização da metodologia o estudo de eventos com uma janela de 15 dias pré e pós evento, baseado em Bootstrap, técnica estatística que realiza simulações para verificar qual a melhor distribuição em que a amostra se enquadra.

A partir de uma base de dados de 11.317 eventos, os resultados indicaram que as distribuições de dividendos e JCP influenciaram o mercado positivamente, já que o retorno acumulado anormal médio $(\overline{C A R})$ das ações foi de $0,70 \%$ no dia do evento (considerando um intervalo de confiança de $95 \%)$.

Outro evento que foi capaz de influenciar positivamente o mercado foi a divulgação de desdobramentos de ações, já que no dia do evento o retorno médio anormal das ações foi de $1,96 \%$, também considerando um intervalo de confiança de $95 \%$, resultados alinhados com o que fora apontado anteriormente por Lamoureux e Poon (1987) e Xiao-Xuan (2003). Os desdobramentos, normalmente, levam a maior liquidez às ações junto ao mercado com a redução nos preços desses papeis.

Além disso, vale ressaltar que os resultados de Freitas e Minardi (2013) com relação aos downgrades de ratings são divergentes dos aqui apresentados uma vez que quando utilizamos os estudos de eventos baseados em Bootstrap não encontramos reações significativas no mercado enquanto que Freitas e Minardi (2013) reportaram uma reação negativa do mercado aos rebaixamentos.

Ademais, apesar de os grupamentos de ações não apresentarem retornos anormais estatisticamente significantes, pode-se inferir graficamente que o retorno das ações em torno deste evento segue uma tendência de baixa, indicando uma sinalização negativa ao mercado.

Como sugestões para pesquisas futuras, indica-se a utilização de outros eventos corporativos para análise do comportamento do mercado, como por exemplo, a data da divulgação das demonstrações financeiras das companhias, que trazem informações relevantes ao mercado, tais como perdas e ganhos. Além disso, os resultados desta pesquisa limitam-se ao período analisado em cada evento e às características inerentes ao mercado acionário brasileiro. Posto isso, pode haver contaminação do índice Ibovespa dada a concentração acionária do mercado brasileiro.

\section{REFERÊNCIAS}

ANGEL, James J. Tick size, share prices, and stock splits. The Journal of Finance, v. 52, n. 2, p. 655-681, 1997.

BALL, Ray; BROWN, Philip. An empirical evaluation of accounting income numbers. Journal of accounting research, p. 159-178, 1968. 
BARTH, Mary E.; BEAVER, William H.; LANDSMAN, Wayne R. The relevance of the value relevance literature for financial accounting standard setting: another view. Journal of accounting and economics, v. 31, n. 1-3, p. 77-104, 2001.

BOLTON, Patrick; FREIXAS, Xavier; SHAPIRO, Joel. The credit ratings game. The Journal of Finance, v. 67, n. 1, p. 85-111, 2012.

BOOTSTRAP, E. B. (1979). Methods: Another look at the Jackknife [J]. Ann. Statist, 7(1), $1-26$.

CAMARGOS, M. A.; BARBOSA, F. V. Estudo de Eventos: Teoria e operacionalização. Caderno de Pesquisa em Administração. São Paulo. v. 10. n.3, p.1-20, jul./set. 2003.

CHOU, Pin-Huang. Bootstrap tests for multivariate event studies. Review of Quantitative Finance and Accounting, v. 23, n. 3, p. 275-290, 2004.

COMIRAN, F. H. (2009). Reação do mercado acionário brasileiro ao grupamento de ações. Dissertação [Mestrado em Administração]. Porto Alegre - Universidade Federal do Rio Grande do Sul.

CORRADO, Charles J. Event studies: A methodology review. Accounting \& Finance, v. 51, n. 1, p. 207-234, 2011.

DAMASCENO, Danilo Luís; ARTES, Rinaldo; MINARDI, Andrea Maria Accioly Fonseca. Determinação da taxa de crédito de empresas brasileiras com uma utilização de índices contábeis. Jornal de Gestão de RAUSP, v. 43, n. 4, p. 344-355, 2008.

DE SENA, Alnio Suamy; DE AZEVEDO FILHO, Azamor Cirne; LUCENA, Wenner Glaucio Lopes. O anúncio de ofertas subsequentes de ações e seu efeito no preço das ações: uma abordagem com base no teste de Pettitt. Revista Universo Contábil, v. 12, n. 1, p. 144$161,2016$.

DVISON, A. C.; HINKLEY, David V.; SCHECHTMAN, Edna. Efficient bootstrap simulation. Biometrika, v. 73, n. 3, p. 555-566, 1986.

FAMA, Eugene F. et al. The adjustment of stock prices to new information. International economic review, v. 10, n. 1, p. 1-21, 1969.

FREIRE, Hércules Vander de Lima et al. Dividendos e lucros anormais: um estudo nas empresas listadas na Bovespa. Revista Contabilidade \& Finanças, v. 16, n. 39, p. 47-67, 2005.

FREITAS, Abner de Pinho Nogueira; MINARDI, Andrea Maria Accioly Fonseca. The impact of credit rating changes in Latin American stock markets. BAR-Brazilian Administration Review, v. 10, n. 4, p. 439-461, 2013.

FROST, Carol Ann. Credit rating agencies in capital markets: A review of research evidence on selected criticisms of the agencies. Journal of Accounting, Auditing \& Finance, v. 22, n. 3, p. 469-492, 2007. 
HARDIN, William G.; LIANO, Kartono; HUANG, Gow-Cheng. REIT stock splits and market efficiency. The Journal of Real Estate Finance and Economics, v. 30, n. 3, p. 297 $315,2005$.

HSUEH, L. Paul; LIU, Y. Angela. Market anticipation and the effect of bond rating changes on common stock prices. Journal of Business Research, v. 24, n. 3, p. 225-239, 1992.

KISGEN, Darren J. Credit ratings and capital structure. The Journal of Finance, v. 61, n. 3, p. 1035-1072, 2006

KOTHARI, S. P.; WARNER, Jerold B. Econometrics of Event Studies, Handbook of Corporate Finance: Empirical Corporate Finance (Handbooks in Finance Series, Elsevier/North-Holland), B. Espen Eckbo (ed.), 2005.

KRAMER, Lisa A. Alternative methods for robust analysis in event study applications. Advances in Investment Analysis and Portfolio Management, v. 8, n. 1, p. 109-132, 2001.

LAMOUREUX, Christopher G.; POON, Percy. The market reaction to stock splits. The journal of finance, v. 42, n. 5, p. 1347-1370, 1987.

LITZENBERGER, Robert H .; RAMASWAMY, Krishna. Os efeitos dos dividendos sobre os efeitos dos impostos sobre as ações ordinárias ou sobre os efeitos das informações? The Journal of Finance, v. 37, n. 2, p. 429-443, 1982.

MACKINLAY, A. Craig. Event studies in economics and finance. Journal of economic literature, v. 35, n. 1, p. 13-39, 1997.

MARTINS, Andressa Iovine; FAMÁ, Rubens. O que revelam os estudos realizados no Brasil sobre política de dividendos? RAE-Revista De Administração De Empresas, v. 52, n. 1, p. 24-39, 2012.

MIAN, G. Mujtaba; SANKARAGURUSWAMY, Srinivasan. Investor sentiment and stock market response to earnings news. The Accounting Review, v. 87, n. 4, p. 1357-1384, 2012.

MOSHIRIAN, Fariborz; NG, David; WU, Eliza. The value of stock analysts' recommendations: Evidence from emerging markets. International Review of Financial Analysis, v. 18, n. 1-2, p. 74-83, 2009.

NETO, Jorge Augusto Novis; SAITO, Richard. Pagamentos de dividendos e persistência de retornos anormais das ações: evidência do mercado brasileiro. Revista de Administração da Universidade de São Paulo, v. 38, n. 2, 2003.

PROCIANOY, Jairo Laser; VERDI, Rodrigo dos Santos. O efeito clientela no mercado brasileiro: será que os investidores são irracionais. Revista Brasileira de Finanças, v. 1, n. 2, p. 217-242, 2003.

PROCIANOY, Jairo Laser; VERDI, Rodrigo S. Dividend clientele, new insights, and new questions: the Brazilian case. RAE eletrônica, v. 8, n. 1, p. 0-0, 2009.

ROSS, Stephen A. et al. Administração financeira. AMGH Editora, 2015. 
SHAH, A.; BALASUBRAMANIAM, V.; BAHURE, V. (2013). Eventstudies: Event study and extreme event analysis. R package version 1.1. https://CRAN.R-

project.org/package=eventstudies

SILVA, Ricardo Luiz Menezes da; NARDI, Paula Carolina Ciampaglia; TONANI, Rafaella. A relevância do dividendo adicional proposto. Revista Contemporânea de Contabilidade, v. 13, n. 29, p. 179-202, 2016.

STOLPER, Anno. Regulation of credit rating agencies. Journal of Banking \& Finance, v. 33, n. 7, p. 1266-1273, 2009.

TEAM, R. Core. A language and environment for statistical computing. R Foundation for Statistical Computing, Vienna, Austria2014. URL: (https://www. R-project. org), 2014.

Tonin, J. M. D. F. (2013). Relação entre income smoohing e ratings em companhias brasileiras de capital aberto. Revista Contabilidade Vista \& Revista, 26(1), 104 - 122.

Valle, M. R. (2002). Mercados de bonds: risco, rating e custo de captação. Revista de Administração da Universidade de São Paulo, 37(2),46-56.

VASSALOU, Maria; XING, Yuhang. Default risk in equity returns. The journal of finance, v. 59, n. 2, p. 831-868, 2004.

XIAO-XUAN, Yang. The Market Reaction To Stock Splits Used as Dividends. Technology and Investment, v. 4, n. 01, p. 42, 2013. 